Rev Inv Vet Perú 2015; 26(4): 676-681

http://dx.doi.org/10.15381/rivep.v26i4.11203

\title{
La Salmonelosis como Factor de Riesgo de Mortinatalidad en Cuyes
}

\author{
Salmonellosis as Risk Factor for Stillbirth in Guinea Pigs \\ Gabriela Ortega O. ${ }^{1,4}$, Ronald Jiménez A. ${ }^{1,5}$, Miguel Ara G. ${ }^{2}$, Siever Morales C. ${ }^{3}$
}

Resumen

Se evaluó la asociación entre casos positivos a aislamiento de Salmonella sp a partir de hisopados vaginales dentro de las 24 horas del parto y la mortinatalidad en una granja de cuyes en Huancayo, Perú. Se trabajó con 258 cuyes siguiendo un diseño de Caso-Control, pareando el tamaño de camada y utilizando como covariables al número de parto y el galpón de procedencia de la reproductora. En el grupo de 'Caso', se incluyeron hembras en cuyo parto hubo por lo menos un mortinato y en el 'Control' hembras con toda la camada viva. El 8.5\% de las reproductoras en el grupo de 'Caso' resultaron positivas a Salmonella $\mathrm{sp}$, y mediante el análisis de regresión logística se estimó una Odds Ratio de 4.32 (95\% de intervalo de confianza, p<0.05) de los casos positivos respecto a los controles. Por otro lado, la causa de muerte en el 91.5\% (118/129) de hembras con mortinatos se debería a otras causas infecciosas o a causas no infecciosas. Los resultados indican que la salmonelosis debe ser considerada como una de las causas de mortinatalidad en cuyes.

Palabras clave: cobayo, mortinato, Salmonella sp, caso-control

\section{Abstract}

The association between positive cases of Salmonella sp isolated from vaginal swabs collected within 24 hours of the parturition and stillbirth in a guinea pig farm in Huancayo, Peru was evaluated. The study included 258 breeding females using a CaseControl design, where litter size was paired between the two groups and parity and number of birth and barn of the breeding female were used as co-variables. In the 'Case' group were included females with at least one stillbirth per litter and in the 'Control'

\footnotetext{
${ }^{1}$ Centro de Investigación IVITA - El Mantaro, Universidad Nacional Mayor de San Marcos, Huancayo, Perú

${ }^{2}$ Laboratorio de Bioquímica, Nutrición y Alimentación Animal, ${ }^{3}$ Laboratorio de Microbiología y Parasitología Veterinaria, Facultad de Medicina Veterinaria, Universidad Nacional Mayor de San Marcos, Lima, Perú

${ }^{4}$ E-mail: gabyortegao@hotmail.com

${ }^{5}$ E-mail: rjimeneza@unmsm.edu.pe
}

Recibido: 21 de enero de 2015

Aceptado para publicación: 29 de mayo de 2015 
group were included females where the entire litter was alive. Results showed that $8.5 \%$ of the breeding females in the 'Case' group were positive to Salmonella sp, and by the logistic regression analysis was estimated an Odds Ratio of 4.32 (95\% confidence interval; $\mathrm{p}<0.05)$ for positive cases in relation to the controls. On the other hand, the cause of death in $91.5 \%(118 / 129)$ of the females with stillbirths was due to other infectious or noninfectious causes. The results show that Salmonella should be considered as a cause of stillbirths in guinea pigs.

Key words: guinea pig, stillbirth, Salmonella sp, case-control

\section{INTRODUCCIÓN}

En el Valle del Mantaro se ha incrementado la crianza comercial del cuy (Cavia porcellus) en los últimos años; sin embargo, todavía existen problemas de orden técnico que afectan su productividad.

La productividad se mide según el índice de productividad anual, el cual representa la cantidad de cuyes aptos para la venta que produce una reproductora en un año ( $R$. Jiménez, Jauja, datos no publicados). Este índice depende de cuatro variables: (i) fertilidad; (ii) tamaño de camada; (iii) número de partos por año; y (iv) viabilidad. Esta última variable expresa la tasa de crías nacidas vivas respecto al total de crías nacidas (dado que la mortinatalidad suele afectar seriamente el número de crías que sobreviven el nacimiento).

La mortinatalidad o mortalidad alrededor del nacimiento puede deberse a procesos fisiológicos o infecciosos. Entre los patógenos causales de mortinatos se encuentran Listeria monocytogenes, Streptococcus pneumoniae, Bordetella brochiseptica, Leptospira sp y Salmonella sp (Ganaway, 1976; Christianson, 1992). Los mecanismos de acción de estos patógenos son diversos, e incluyen infección directa, daño a la placenta y severo compromiso sistémico de la madre (Goldenberg y Thompson, 2003).

Existen serotipos de Salmonella enterica capaces de producir natimortos y abortos en mamíferos, ejemplo de ello es la Salmonella Abortusovis y las Salmonella Montevideo y Dublin (NADIS, 2003; CFSPH, 2005). Asimismo, se han descrito casos de natimortos en mujeres que se infectaron al consumir alimento contaminado con Salmonella typhi (Dean y Kendall, 2006).

En la crianza comercial de cuyes en el valle del Mantaro, Perú, la tasa de mortinatalidad se encuentra entre 15 y $18 \%$ (Jiménez y Huamán, 2010), siendo la salmonelosis la enfermedad de mayor prevalencia, cuyas pérdidas económicas llegan hasta $53 \%$ por morbilidad y $95 \%$ por mortalidad (Morales et al., 2007). El presente estudio pretendió determinar el grado de riesgo de la salmonelosis en la mortinatalidad en cuyes.

\section{Materiales y Métodos}

El trabajo se llevó a cabo entre los meses de febrero a mayo de 2008 en la granja de cuyes y en el Laboratorio de Microbiología del Centro de Investigación IVITA, Facultad de Medicina Veterinaria, Universidad Nacional Mayor de San Marcos, ubicados en el distrito de El Mantaro, provincia de Jauja, región Junín, Perú, a una altitud de 3320 m, con una temperatura ambiental que oscila entre +19.9 y $-1.8^{\circ} \mathrm{C}$ y una precipitación pluvial promedio anual de $770 \mathrm{~mm}$.

Los cuyes provinieron de una población de reproductoras mejoradas por selección en ganancia de peso y conformación corporal. 
La granja reportó en 2007 una tasa de mortalidad de $2.5 \%$ mensual, siendo la salmonelosis responsable del $20 \%$ de estas muertes.

El tamaño de muestra se estimó empleando la fórmula para la comparación de dos proporciones (Pita Fernández, 1996). Se trabajó con un tamaño de muestra de 129 cuyes para cada grupo, de un total de 480 reproductoras distribuidas en los cinco galpones de la granja.

Se seleccionaron 258 animales de cinco galpones que registraban partos con y sin ocurrencia de nacidos muertos. De estos, se tuvo hembras de 1, 2, 3 y más de 3 partos, cada grupo con 86 animales. El número de reproductoras por galpón fue de 51, 56, 76, 39 y 36 para los galpones 1, 2, 3, 4 y 5, respectivamente. En los galpones 1, 2, 4 y 5, las reproductoras tuvieron un manejo individualizado desde una semana antes del parto hasta el destete, y con una mejor disponibilidad de alimento; mientras que en el galpón 3, el manejo de las reproductoras fue en grupos de 7 cuyes por poza con cierto grado de competencia por el alimento. El promedio de tamaño de camada del total de animales muestreados fue de 3.58 , con rango de 1 a 6 . En el grupo de reproductoras con mortinatos $(n=129)$, el promedio de mortinatos fue de 1.82 , con rango de 1 a 5 .

Se tomó una muestra de hisopado vaginal de cada hembra de ambos grupos dentro de las 24 horas del parto. Las muestras fueron rotuladas y llevadas al laboratorio en medio de transporte Stuart. El hisopado fue resuspendido en $1 \mathrm{ml}$ de caldo tripticasa soya por $24 \mathrm{~h}$ y cultivado en caldo Selenito a $37^{\circ} \mathrm{C}$ durante $24 \mathrm{~h}$. Luego se procedió a sembrar por agotamiento en placas de agar Salmonella-Shigella (SS) e incubado a $37^{\circ} \mathrm{C}$ durante $24 \mathrm{~h}$. La identificación bacteriana se realizó mediante coloración Gram y pruebas de catalasa y oxidasa a las colonias compatibles con Salmonella sp. Las colonias que resultaron ser bacilos gramnegativos, catalasa positivo y oxidasa negativo fueron sometidas a las pruebas bioquímicas de Indol, Citrato, Ureasa, $\mathrm{H}_{2} \mathrm{~S}$, Movilidad, Lisina, Lactosa, Sacarosa y Gas de Glucosa (Caffer y Terragno, 2001; Terragno et al., 2003).

Se empleó el diseño epidemiológico Caso-Control con $n$ fijo, donde 'Caso' se denomina al parto de una cuy hembra con al menos un mortinato en su camada, y 'Control' al homólogo pero con toda la camada viva. Además, se pareó el tamaño de camada y se consideraron como covariables el número de parto y el galpón de procedencia de la reproductora. Los mortinatos se confirmaron mediante la prueba de docimasia pulmonar hidrostática (Romo, 2000). Se estimó el Odds Ratio (OR) con un intervalo de confianza (IC) de $95 \%$, empleando el modelo de regresión logística del paquete estadístico SAS 8.1 (SAS Institute, 1990).

\section{Resultados y Discusión}

Se identificaron $11(8.5 \%)$ casos y 3 (2.3\%) controles positivos a Salmonella spp (Cuadro 1), siendo el OR (4.32) estadísticamente significativo $(\mathrm{p}<0.05)$ (Cuadro 2), determinando que la mortinatalidad está influenciada por este patógeno. El tamaño de camada, número de parto y galpón de procedencia no fueron estadísticamente importantes en la ocurrencia de mortinatos (Cuadro 2).

El 2.3\% (3/129) de las cuyes que no presentaron problemas de muerte de gazapos durante el parto resultaron positivas al aislamiento (Cuadro 1). Se presume que estos animales estarían comportándose como portadores asintomáticos; es decir, no manifestaron la enfermedad en términos de pérdidas de crías a pesar del relajamiento de la inmunidad por el parto. Esto es un dato de importancia que debe considerarse en la epidemiología y control de la enfermedad (Suárez y Mantilla, 2000; Fica et al., 2001). 
Cuadro 1. Relación entre mortinatalidad en camadas de cuyes y aislamiento de Salmonella $\mathrm{sp}$ a partir de hisopados vaginales dentro de las 24 horas del parto

\begin{tabular}{lccc}
\hline \multirow{2}{*}{ Camada de gazapos por reproductora } & \multicolumn{2}{c}{ Aislamiento de Salmonella sp } & \multirow{2}{*}{ Total } \\
\cline { 2 - 3 } & Positivos & Negativos & \\
\hline Caso (al menos un mortinato) & 11 & 118 & 129 \\
Control (todos vivos) & 3 & 126 & 129 \\
\hline Total & 14 & 244 & 258 \\
\hline
\end{tabular}

Cuadro 2. Análisis de regresión logística para las variables asociadas a mortinatos en cuyes reproduc toras de la granja del IVITA - El Mantaro (2008)

\begin{tabular}{|c|c|c|c|}
\hline Variables ${ }^{1}$ & Odds ratio & IC $95 \%^{2}$ & $P$ \\
\hline \multicolumn{4}{|c|}{ Aislamiento de Salmonella sp } \\
\hline Positivo & 4.32 & $1.15-16.29$ & 0.03 \\
\hline \multicolumn{4}{|l|}{ Número de parto } \\
\hline $2^{\text {do }}$ y $3^{\text {ro }}$ & 0.64 & $0.35-1.18$ & 0.16 \\
\hline Más de 3 & 1.13 & $0.39-3.27$ & 0.83 \\
\hline \multicolumn{4}{|l|}{ Galpón } \\
\hline 1 & 1.06 & $0.49-2.26$ & 0.89 \\
\hline 2 & 1.12 & $0.54-2.34$ & 0.76 \\
\hline 4 & 1.00 & $0.32-3.11$ & 0.99 \\
\hline 5 & 0.84 & $0.29-2.46$ & 0.75 \\
\hline
\end{tabular}

${ }^{1}$ Se tomaron como referencias el aislamiento negativo a Salmonella sp, el primer parto y el gal pón tres

${ }^{2}$ Intervalo de confianza

Un resultado importante de enfatizar es que no se aisló Salmonella sp en el $91.5 \%$ $(118 / 129)$ de hembras que presentaron al menos un mortinato. Esto indica que la causa de la muerte de los gazapos podría deberse a otros patógenos como Bordetella bronchiseptica, Listeria monocytogenes, Streptococcus pneumoniae y Leptospira $\mathrm{sp}$ (Ganaway, 1976; Christianson, 1992; Smith et al., 2003), o estar asociada a factores no infecciosos como tamaño de camada, núme- ro de partos por año, incrementos de temperatura ambiental y toxemia de la preñez (Wagner, 1976; Christianson, 1992); esta última relacionada básicamente al tipo de alimentación (Cheeke, 1995).

Los casos positivos a salmonelosis solo representaron el 8.5\% (11/129) del total de casos con mortinatos. Reportes en ovejas señalan niveles superiores; por ejemplo Astorga et al. (2008) reportaron $16 \%$ de 
mortalidad perinatal en ovejas causada por Salmonella Indiana, en un brote que incluyó abortos en el último tercio de gestación, y mortinatos y corderos no viables que fallecían en pocas horas; asimismo, Mearns (2007) señala que puede ocurrir hasta un 60\% de abortos y mortinatos en brotes de Salmonella var abortusovis en rebaños de ovejas cuando el patógeno ingresa por primera vez en la majada.

Los resultados del presente estudio permiten concluir que la salmonelosis es un factor de riesgo de mortinatalidad en la crianza de cuyes

\section{Agradecimientos}

Los autores agradecen a INCAGRO (Fondo de Desarrollo Estratégico) por el financiamiento del estudio a través del subproyecto «Desarrollo y evaluación de reproductores para maximizar la producción de cuyes de carne» con contrato 2006-00287AG- INCAGRO/FDSE.

\section{Literatura Citada}

1. Astorga RJ, Luque I, León J, García I, Echeita A. 2008. Brote de mortalidad perinatal en ovejas de raza Lacaune asociado a Salmonella Indiana. Prod Anim N. ${ }^{\circ}$ 249: 6-13.

2. Caffer M, Terragno R. 2001. Manual de procedimientos para la caracterización de Salmonella. Buenos Aires, Argentina: Ministerio de Salud, Instituto Nacional de Enfermedades Infecciosas ANLIS «Dr. Carlos G. Malbrán». 37 p.

3. [CFSPH] Center for Food Security and Public Health. 2005. Salmonella Abortusovis: paratyphoid abortion. College of Veterinary Medicine Iowa State University. [Internet]. Disponible en: http://www.cfsph.iastate.edu/Factsheets/pdfs/salmo-nellaabor-tusovis.pdf

4. Cheeke PR. 1995. Alimentación y nutrición del conejo. Zaragoza: Acribia. 429 p.
5. Christianson WT. 1992. Stillbirths, mummies, abortions and early embryonic death. Vet Clin North Am Food Anim Pract 8: 623-639.

6. Dean J, Kendall P. 2006. Food safety during pregnancy. Colorado State University. N. ${ }^{\circ} 9.372$. [Internet]. Disponible en: http://www.ext.colostate.edu/ pubs/FOODNUT/09372.html

7. Fica A, Alexandre M, Prat S, Fernández A, Fernández J, Heitmann I. 2001. Cambios epidemiológicos de las salmonelosis en Chile. Desde Salmonella typhi a Salmonella enteritidis. Rev Chil Infectol 18: 85-93. doi: 10.4067/S0716-10182001000200002

8. Ganaway JR. 1976. Bacterial, Mycoplasma, and Rickettsial diseases. En: Wagner JE, Manning PJ (eds). The biology of guinea pig. USA: Academic Press. p 121-135.

9. Goldenberg R, Thompson C. 2003. The infectious origins of stillbirth. Am J Obstet Gynecol 189: 861-873. doi: 10.1067/S0002-9378(03)00470-8

10. Jiménez R, Huamán A. 2010. Manual para el manejo de reproductores híbridos especializados en producción de carne. El Mantaro, Perú: INCAGROACRICUCEN-UNMSM. $175 \mathrm{p}$.

11. Mearns R. 2007. Other infectious causes of abortion. En: Aitken ID (ed). Diseases of sheep. $4^{\text {th }}$ ed. UK: Blackwell Publishing. p 127-130.

12. Morales S, Mattos J, Calle S. 2007. Efecto de la muña (Satureja parvifolia) en la dinámica de la infección por Salmonella enterica en cobayos. En: XXX Reunión ALPA. Cusco: Asociación Latinoamericana de Producción Animal.

13. [NADIS] National Animal Disease Information Service. 2003. NADIS Sheep Disease Bulletin: Salmonella abortion in ewes. [Internet]. Disponible en: http://www.qmscotland.co.uk/ a n a 1 y s i s / d o w n lo a d s / Salmonella\%20abortion\%20in\%20ewes.pdf

14. Pita Fernández S. 1996. Determinación del tamaño muestral. Cad Aten Primaria 3: 138-141. 
15. Romo O. 2000. El nacimiento. Comienzo de la personalidad legal. En: Romo O (ed). Medicina legal. Elementos de ciencias forenses. Chile: Ed Jurídica de Chile. p 230-231.

16. SAS Institute. 1990. SAS/STAT User's Guide, v. $6.4^{\text {th }}$ ed. Vol 1. Cary, NC: SAS Institute Inc. 943 p.

17. Smith MA, Takeuchi K, Backett RE, McClure HM, Raybourne RB, Williams KM, Babu MS, et al. 2003. Nonhuman primate model for listeria monocytogenes-induced stillbirth. Infect Immun 71: 1574-1579. doi: 10.1128/ IAI.71.3.1574-1579.2003

18. Suárez MC, Mantilla JR. 2000. Presencia de salmonella serovariedad
Enteritidis en productos de origen avícola y su repercusión en salud pública. Iatreia 13: 237-245.

19. Terragno $R$, Caffer $M$, Bruno $S$, Binsztein N. 2003. Salmonella: aislamiento, identificación y serotipificación. En: Manual de procedimientos. Argentina: Instituto Nacional de Enfermedades Infecciosas - ANLIS «Dr. Carlos G. Malbrán». $46 \mathrm{p}$.

20. Wagner JE. 1976. Miscellaneous disease conditions of guinea pigs: IV. Urogenital system. En: Wagner JE, Manning PJ (eds). The biology of guinea pig. USA: Academic Press. p 229-230. 